\title{
DENSIDADE DE ESTOCAGEM NA ALEVINAGEM DE TILÁPIA-DO-NILO EM TANQUE-REDE
}

\author{
Henrique Maeda, ${ }^{1}$ Paulo Cesar Silva, ${ }^{2}$ Raquel Priscilla de Castro Oliveira, ${ }^{3}$ Marília da Silva Aguiar, ${ }^{4}$ \\ Delma Machado Cantisani Pádua, ${ }^{5}$ Nádia Pales Machado, ${ }^{6}$ Valéria Rodrigues ${ }^{6}$ E \\ Rene Henrique da Silva ${ }^{7}$
}

1. Mestrando em Ciência Animal da Escola de Veterinariária da Universidade Federal de Goiás, Goiânia, GO

2. Professor doutor do Departamento de Produção Animal e responsável pelo Setor de Piscicultura da EV/UFG

3. Doutoranda em Ciência Animal da EV/UFG - E-mail: kekelgyn@yahoo.com.br

4. Pesquisadora colaboradora EV/UFG

5. Professora do Departamento de Zootecnia, PUC-GO

6. Acadêmicos do Curso de Medicina Veterinária da EV/UFG

7. Acadêmico de Zootecnia da UEG.

\section{RESUMO}

Este estudo foi desenvolvido com o intuito de avaliar densidades de estocagem alevinagem de tilápia nilótica (Oreochromis niloticus) realizado em tanques-rede, durante 43 dias. Utilizaram-se quinze tanques-rede de $0,18 \mathrm{~m}^{3}$, instalados em viveiro com $80 \%$ de troca diária da água. As densidades testadas foram: $700,1.000$ e 1.300 alevinos $/ \mathrm{m}^{3}$ com peso médio inicial de $4,77 \pm 0,29 \mathrm{~g}$. Avaliaram-se as seguintes variáveis de desempenho produtivo: sobrevivência; peso final; comprimento final; biomassa final; conversão alimentar aparente; ganho de peso; ganho de peso diário; taxa de eficiência proteica; taxa de crescimento específico e fator de condição. Utilizou-se análise de variância para avaliação dos resultados e quando significativo aplicou-se teste Tukey a 5\%. O tratamento com maior densidade de estocagem diferiu significativamente $(\mathrm{p}<0,05)$ do tratamento com menor densidade em relação ao peso final, biomassa final, ganho de peso, ganho de peso diário e taxa de crescimento específico. As demais variáveis não apresentaram diferenças significativas entre os tratamentos ( $p>0,05)$. Concluiu-se que, nestas condições experimentais, as melhores densidades de estocagem na segunda alevinagem em tanques-rede são de 700 a 1.000 alevinos $/ \mathrm{m}^{3}$.

PALAVRAS-CHAVES: Desempenho produtivo, gaiolas, Oreochromis niloticus, sistema intensivo.

\section{ABSTRACT}

\section{STOCKING DENSITY OF NILE TILAPIA (Oreochromis niloticus) FINGERLINGS IN NETCAGES}

The experiment evaluated the stocking density of Nile tilapia (Oreochromis niloticus) fingerling in netcages for 43 days. Fifteen $180 \mathrm{~L}$ netcages were used as experiment units, in a pond with $80 \%$ water renovation. The tested stocking densities were 700 fingerlings $/ \mathrm{m}^{3} ; 1,000$ fingerlings $/ \mathrm{m}^{3}$ and 1,300 fingerlings/ $\mathrm{m}^{3}$. The mean initial weight of the fingerlings was $4.77 \pm 0.29$ g. The following parameters were analyzed: mortality rate; final weight; final length; final biomass; feed conversion rate; weight gain; daily weight gain; protein efficiency rate; specific growth rate and condition factor. Variance analyzes and Tukey test at 5\% were applied to compare the different treatments. Treatment with greater stocking density differed significantly from treatment with lower density regarding final weight, final biomass, weight gain, daily weight gain and specific growth rate. The other parameters did not present significant differences among the treatments $(\mathrm{p}>0.05)$. Under these experimental conditions, the best stocking densities for cages were 1,000 to 1,300 fingerlings $/ \mathrm{m}^{3}$.

KEYWORDS: Cages, growth performance, intensive system, Oreochromis niloticus. 


\section{INTRODUÇÃO}

A tilápia-do-nilo (Oreochromis niloticus) destaca-se por apresentar rápido crescimento, alta prolificidade e grande capacidade de filtrar partículas do plâncton. Tolera condições ambientais adversas, como baixo oxigênio dissolvido $(1,0 \mathrm{mg} / \mathrm{L})$, sendo que, em viveiros de recria, alevinos pesando entre 10 e 25 g podem suportar concentrações de oxigênio dissolvido entre 0,4 e $0,7 \mathrm{mg} / \mathrm{L}$ por três a cinco horas e quatro manhãs seguidas (KUBITZA, 2000); suportam ainda altos níveis de amônia não ionizada $(2,4$ a $3,4 \mathrm{mg} / \mathrm{L})$ e $\mathrm{pH}$ entre cinco e onze (WATANABE et al., 2003).

Uma importante fase da criação de peixes é a segunda alevinagem, que tem como objetivo aumentar o peso dos alevinos de 2,0 g para 20 ou $30 \mathrm{~g}$, garantindo, assim, maior sobrevivência, por estarem mais fortes e resistentes (PROENÇA \& BITTENCOURT, 1994; CASACA \& TOMAZELLI JR., 2001).

Segundo MAEDA et al. (2006), trabalhos científicos desenvolvidos especificamente sobre tecnologia de segunda alevinagem são importantes, porque se trata de uma fase a ser incorporada no pacote tecnológico fornecido ao setor produtivo. Para GALVÃO (2008), ao adotar a técnica da segunda alevinagem, animais com $6,0 \mathrm{~cm}$, o produtor pode reduzir consideravelmente o custo de produção.

De acordo com ONO \& KUBITZA (1999) e CYRINO et al. (2002), tanques-rede caracterizamse por ser estrutura de baixo custo e de fácil implantação em comparação com outras modalidades de produção, pois possibilitam o aproveitamento de represas, lagos e outros corpos hídricos já existentes. Apresentam algumas vantagens, como menor investimento inicial, e possibilitam controle na alimentação e manejo dos peixes, quando comparado a outros sistemas.

Em contrapartida, oferecem algumas desvantagens, como risco de rompimento da tela ou rede e consequente perda dos animais, impossibilidade de instalação em águas de baixa qualidade e dependência de ração completa e enriquecida (WATANABE et al., 2003). Além da possibilidade de manifestação de doenças, em virtude de maior estresse (BARCELLOS et al., 2004).
Segundo ONO \& KUBITZA (1999), os peixes confinados apresentam acesso restrito ao alimento natural disponível no ambiente. Assim, a ração utilizada no cultivo em tanques-rede deve ser nutricionalmente completa, suprindo todas as necessidades dos peixes em nutrientes. FURUYA et al. (2000) consideraram que ração com $30 \%$ de proteína digestível (equivalente a $32 \%$ de $\mathrm{PB}$ ) atende às exigências de alevinos de tilápia-do-nilo.

Ainda são poucos os trabalhos científicos relacionados à tecnologia de segunda alevinagem, especialmente em tanques-rede. Com isso, este trabalho foi desenvolvido com o objetivo de avaliar densidades de estocagem para a obtenção do alevino de tilápia-do-nilo, em gaiolas.

\section{MATERIAL E MÉTODOS}

A pesquisa foi realizada no Setor de Piscicultura do Departamento de Produção Animal da Escola de Veterinária da Universidade Federal de Goiás, no período de 17 de novembro a 29 de dezembro de 2003, totalizando 43 dias.

Utilizaram-se 2.700 alevinos de tilápia-donilo, revertidos para macho, com peso médio inicial de 4,77 $\pm 0,29 \mathrm{~g}$ e comprimento médio inicial de $5,19 \pm 0,54 \mathrm{~cm}$. A biometria foi realizada apenas no início e no final do experimento, por amostragem, evitando, assim, estresse dos peixes. As pesagens foram realizadas em pool. No início, pesaram-se onze lotes antes da distribuição dos alevinos nas unidades experimentais, sendo que cada lote possuía dez alevinos escolhidos ao acaso. Ao final da pesquisa, foram pesados dez lotes, cada um com cinco animais, perfazendo uma amostra de cinquenta peixes de cada tanque-rede.

O arraçoamento foi com ração comercial extrusada, fornecida pela Rações VB, ad libitum, três vezes ao dia (8:00, 12:00 e 16:00 horas). Procedeuse à análise bromatológica da ração fornecida, no Laboratório de Nutrição Animal da Universidade Federal de Goiás, encontrando-se as seguintes composições: $36 \% \mathrm{~PB} ; 3,5 \% \mathrm{EE} ; 12,8 \% \mathrm{MM} ; 2,0 \% \mathrm{Ca}$; $1,0 \% \mathrm{P}$ e $2,0 \mathrm{~mm}$ de diâmetro.

As unidades experimentais foram constituídas por quinze tanques-rede, de malha multifilamento de cinco milímetros e $180 \mathrm{~L}$ de volume útil $(0,60$ 
$\left.\times 0,60 \times 0,50 \mathrm{~m}=0,18 \mathrm{~m}^{3}\right)$, instalados em viveiro coberto com tela antipássaro, com $360 \mathrm{~m}^{2} \mathrm{e} 1,5 \mathrm{~m}$ de profundidade e submetido à vazão de abastecimento de cinco litros por segundo (proporcional à troca de $80 \%$ de água por dia). Manteve-se espaçamento entre eles de aproximadamente um metro. Cada tanquerede era dotado de comedouro circular, com tela de um milímetro, para evitar perda de ração. Os peixes foram distribuídos aleatoriamente nos seguintes tratamentos: 700 alevinos $/ \mathrm{m}^{3}$ (126/tanque-rede); 1.000 alevinos $/ \mathrm{m}^{3}$ (180/tanque-rede); 1.300 alevinos $/ \mathrm{m}^{3}$ (234/tanque-rede).

Os seguintes parâmetros físicos-químicos de qualidade de água foram aferidos semanalmente com kit de análise química de água comercial da marca Alfakit: oxigênio dissolvido; pH; amônia tóxica; nitrito, alcalinidade, transparência e diariamente a temperatura, com termômetro de bulbo de mercúrio.

Estudaram-se as seguintes características de desempenho: peso final; ganho de peso; ganho de peso diário (ganho de peso/duração em dias da pesquisa); biomassa inicial e final; conversão alimentar aparente (consumo de ração/ganho em biomassa); sobrevivência; crescimento; taxa de eficiência proteica [ganho em biomassa/(consumo de ração $\mathrm{x} \%$ de proteína)]; taxa de crescimento específico [ $(\log$ peso final - log peso inicial/duração em dias da pesquisa) e fator de condição [peso final/(comprimento final) $)^{3}$ x 100 .

Ao final do experimento constatou-se que um tanque-rede apresentava furos na malha, o que possibilitou a fuga de vários alevinos. Esse tanque-rede foi considerado parcela perdida, ficando o experimento com quatorze unidades experimentais.

O delineamento experimental utilizado foi o inteiramente casualizado, com três tratamentos e cinco repetições. Submeteram-se os resultados à análise de variância, sendo complementados pelo teste de comparação de médias Tukey a 5\% (BANZATTO \& KRONKA, 1995).

\section{RESULTADOS E DISCUSSÃO}

As médias da temperatura da água, pela manhã à a tarde, foram respectivamente de $26,9 \pm 2,39^{\circ} \mathrm{C} \mathrm{e}$ $29,9 \pm 3,34^{\circ} \mathrm{C}$, aproximando-se do conforto térmico para tilápia-do-nilo. O teor de oxigênio dissolvido manteve-se em níveis adequados para a produção de tilápias (KUBITZA, 2000), mas decresceu ao longo do experimento, chegando a $3,3 \mathrm{mg} / \mathrm{L}$ no último dia.

Os níveis de $\mathrm{pH}(6,5$ a 7,0), amônia tóxica $(0,0$ a $0,003 \mathrm{mg} / \mathrm{L})$, nitrito $(0,0 \mathrm{mg} / \mathrm{L}$ - valor não detectado pelo kit) e alcalinidade (26 a $46 \mathrm{mg} / \mathrm{L}$ ) ficaram dentro dos valores recomendados por KUBITZA (1999) e WATANABE et al. (2003) e semelhantes aos obtidos por AYROZA et al. (2000). A transparência da água foi de $65 \mathrm{~cm}$, provavelmente em virtude da alta vazão de água adotada para o viveiro.

Os resultados obtidos para os parâmetros de desempenho produtivo estão expressos na Tabela 2.

O comprimento final não foi afetado pelos tratamentos, mas houve apenas tendência de redução com o aumento da densidade de estocagem, possivelmente por causa da competição por espaço e piora da qualidade da água no interior de cada tanque-rede. MAEDA (2004) e MAEDA et al. (2006) observaram resultados semelhantes.

Encontrou-se diferença estatística entre os tratamentos para peso final. $\mathrm{O}$ tratamento intermediário foi estatisticamente semelhante ao que apresentava maior e menor densidade, porém estes mostraram-se estatisticamente diferentes entre si. Houve redução no peso final com o aumento da densidade de estocagem, possivelmente pelos mesmos motivos comentados anteriormente, concordando com MAEDA (2004) e MAEDA et al. (2006).

A biomassa final do tratamento intermediário foi estatisticamente semelhante aos que apresentavam maior e menor densidade, que, por outro lado, diferenciaram entre si. MAEDA (2004) não encontrou diferenças de biomassa entre as densidades, apenas tendência de aumento acompanhando o aumento da densidade. Já MAEDA et al. (2006) verificaram que o tratamento com menor densidade de estocagem apresentou biomassa final significativamente menor que os outros dois tratamentos, e também observaram tendência de aumento com o incremento da densidade.

Os valores de conversão alimentar aparente (CAa) não diferiram estatisticamente entre si e estão próximos dos recomendados por ONO \& 
KUBITZA (1999) para tilápia em tanque-rede (1,4 a 1,8$)$, sendo bem menores do que os encontrados por HUCHETTE \& BEVERIDGE $(2003)(3,18)$, para tilápias alimentadas com ração e estocadas na densidade de $600 \mathrm{~g} / \mathrm{m}^{3}$ em tanque-rede.

No entanto, MAEDA (2004) e MAEDA et al. (2006) observaram índices menores do que os deste estudo e não encontraram diferenças significativas entre as três densidades estudadas. Os altos índices encontrados neste estudo podem ser atribuídos à maior perda eventual de ração para fora dos tanquesrede.

O fato de a CAa, em alguns casos, ser menor nas altas densidades de estocagem pode ser reflexo do efeito de grupo, isto é, algumas espécies apresentam melhor ingestão e aproveitamento de alimentos quando estocadas em altas densidades, em virtude da redução das agressões e da competitividade da população confinada (CARACIOLO et al., 2000). Segundo WALLACE \& KOLBEINSHAUN (1988), quando o alimento não é limitante e a densidade de estocagem é alta, o surgimento de hierarquias na população pode ser reduzido.
Quanto ao ganho de peso e ganho de peso diário, os tratamentos com menor densidade e com maior densidade foram estatisticamente diferentes, sendo semelhantes ao tratamento intermediário. Com o aumento da densidade de estocagem, o ganho de peso e o ganho de peso diário diminuíram, provavelmente em função do aumento da concorrência pelo alimento, concordando com SILVA et al. (1994) e BARCELLOS et al. (2004), mas discordando dos experimentos de MAEDA (2004) e MAEDA et al. (2006), em que não foram observadas diferenças entre as densidades, possivelmente por não terem atingido as lotações máximas da segunda alevinagem, respectivamente, nos sistemas intensivo tradicional e raceway, este último por causa da alta mortalidade.

Não se encontraram diferenças significativas entre os tratamentos para a taxa de eficiência proteica. Valores superiores foram encontrados também por MAEDA (2004) e MAEDA et al. (2006), respectivamente, 2,61 a 2,65 e 1,80 a 2,23, utilizando a mesma ração deste estudo $(36 \% \mathrm{~PB})$, e por FURUYA et al. (2000), 2,49, em tanques submetidos à troca total de água em 16,6 h.

TABELA 1. Médias dos parâmetros de desempenho produtivo e coeficientes de variação obtidos na segunda alevinagem de tilápia nilótica em tanques-rede

\begin{tabular}{|c|c|c|c|c|}
\hline Parâmetro & 700 alevinos $/ \mathrm{m}^{3}$ & 1.000 alevinos $/ \mathrm{m}^{3}$ & 1.300 alevinos $/ \mathrm{m}^{3}$ & $\mathrm{CV}(\%)$ \\
\hline Sobrevivência (\%) & $90,48 \mathrm{a}$ & $86,67 \mathrm{a}$ & $90,95 \mathrm{a}$ & 91,9 \\
\hline Comprimento final (cm) & $10,16 \mathrm{a}$ & $9,8 \mathrm{a}$ & $9,70 \mathrm{a}$ & 7,8 \\
\hline Peso final (g) & $41,54 \mathrm{a}$ & $38,53 \mathrm{ab}$ & $31,14 \mathrm{~b}$ & 14,3 \\
\hline Biomassa final (g) & $4.735,66 \mathrm{~b}$ & $5.934,80 \mathrm{ab}$ & $6.581,10 \mathrm{a}$ & 14,1 \\
\hline Conversão alimentar aparente & $1,75 \mathrm{a}$ & $1,70 \mathrm{a}$ & $1,79 \mathrm{a}$ & 11,8 \\
\hline Ganho de peso (g) & 36,76 a & $33,75 \mathrm{ab}$ & $26,36 \mathrm{~b}$ & 16,5 \\
\hline Ganho de peso diário (g/dia) & $0,85 \mathrm{a}$ & $0,78 \mathrm{ab}$ & $0,61 \mathrm{~b}$ & 16,5 \\
\hline Taxa de eficiência proteica $(\mathrm{g} / \mathrm{g})$ & $1,59 \mathrm{a}$ & $1,65 \mathrm{a}$ & $1,57 \mathrm{a}$ & 11,9 \\
\hline Taxa de cresc. específico (\%/dia) & $2,18 \mathrm{a}$ & $2,10 \mathrm{ab}$ & $1,88 \mathrm{~b}$ & 7,0 \\
\hline Fator de condição & $3,97 \mathrm{a}$ & $4,09 \mathrm{a}$ & $3,42 \mathrm{a}$ & 12,2 \\
\hline
\end{tabular}

Médias seguidas de letras iguais, na mesma linha, não diferem entre si pelo teste de Tukey $(P>0,05)$ 
Em relação à taxa de crescimento específico, o tratamento intermediário foi estatisticamente semelhante ao de maior e menor densidade e estes se diferenciaram entre si. Estes valores foram bem superiores ao encontrado por HUCHETTE \& BEVERIDGE (2003) $(0,88 \%$ /dia $)$. Em pesquisas desenvolvidas no mesmo Setor, não se verificaram diferenças entre os tratamentos, sendo os dados de MAEDA (2004) superiores e MAEDA et al. (2006) inferiores aos deste estudo. Nos três experimentos, observou-se queda da taxa de crescimento específico com o aumento da densidade de estocagem.

Os valores do fator de condição foram estatisticamente semelhantes entre si e próximos aos obtidos por SOLIMAN et al. (2000a), utilizando ração com $36 \%$ de PB e ligeiramente melhores do que os obtidos por SOLIMAN (2000b). Nos experimentos de MAEDA (2004) e MAEDA et al. (2006), os dados foram estatisticamente semelhantes e próximos aos obtidos neste experimento.

\section{CONCLUSÕES}

Nas condições experimentais explicitadas para segunda alevinagem em tanques-rede, as densidades de estocagem estudadas mostraram-se no limite de suporte, já que os valores dos parâmetros produtivos apresentaram tendência de piora com o aumento da densidade, apesar de permanecerem dentro de limites aceitáveis. Concluise, portanto, que as melhores densidades para a segunda alevinagem podem ser de 700 a 1.000 alevinos $/ \mathrm{m}^{3}$.

\section{AGRADECIMENTOS}

Às Rações VB, pelo fornecimento das rações experimentais, e ao $\mathrm{CNPq}$, pelo fornecimento das bolsas de estudo.

\section{REFERÊNCIAS}

BANZATTO, D. A.; KRONKA, S. N. Experimentação agrícola. 3. ed. Jaboticabal: FUNEP, 1995. 247 p.

BARCELlOS, L. J. G.; KREUTZ, L. C.; QUEVEDO, R. M.; FIOREZE, I.; CERICATO, L.; SOSO, A. B.; FAGUNDES, M.; CONRAD, J.; BALDISSERA, R. K.; BRUSCHI, A.; RITTER, F.
Nursering rearing of jundiá, Rhamdia quelen (Quoy \& Gaimard) in cages: cage type, stocking density and stress response to confinement. Aquaculture, v. 232, issues 1-4, p. 383-394, 2004.

BRAGA, F. M. S. Estudo entre fator de condição e relação peso/ comprimento para alguns peixes marinhos. Revista Brasileira de Biologia, v. 46, n. 2, p. 339-346, 1986.

CASACA, J. M.; TOMAZELLI JR, O. Produção de alevino II. Florianópolis: Epagri, 2001. 29 p. [Boletim Técnico, 115].

FURUYA, W. M.; HAYASHI, C.; FURUYA, V. R. B.; SOARES, C. M. Exigências de proteína digestível para alevinos de tilápiado-nilo (Oreochromis niloticus). Revista Brasileira de Zootecnia, v. 29, n. 6, p. 1912-1917, 2000. [Suplemento 1].

GALVÂO, I. Contribuição para optimizaçâo da tenocologia de propagação artificial de Colossomas, tambaqui (Colossoma macropomum) e pacu (Colossoma mitrei). Disponível em: $<$ http:// www.fao.org/docrep/field/003/AB491S/AB491S03.htm>Acesso em: 22 ago. 2008.

HUCHETTE, S. M. H.; BEVERIDGE, M. C. M. Tecnical and economical evaluation of periphyton-based cage culture of tilapia (Oreochromis niloticus) in tropical freshwater cages. Aquaculture, v. 218, p. 219-234, 2003.

KUBITZA, F. Qualidade da água na produção de peixes. 3. ed. Jundiaí: F. Kubitza, 1999. 97 p.

KUBITZA, F. Tilápia: tecnologia e planejamento na produção comercial. 1. ed. Jundiaí: F. Kubitza, 2000. 285 p.

MAEDA, H.; SILVA, P. C.; AGUIAR, M. S.; PADUA, D. M. C.; OLIVEIRA, R. P. C.; MACHADO, N. P.; RODRIGUES, V.; SILVA, R. H. Efeito da densidade de estocagem na segunda alevinagem de tilápia nilótica (Oreochromis niloticus), em sistema raceway. Ciência Animal Brasileira, v. 7, n. 3, p. 265-272, jul.-set. 2006.

ONO, E. A; KUBITZA, F. Cultivo de peixes em tanques-rede. 2. ed. Jundiaí: F. Kubitza, 1999. 68 p.

PROENÇA, C. E. M.; BITTENCOURT, P. R. L.; Manual de piscicultura tropical. Brasília: IBAMA, 1994. 195 p.

WALLACE, J. C.; KOLBEINSHAUN, A. G. The effect of size granding on subsequent growth in fingerling Artic Charr, Salvelinus alpinus (L), Aquaculture, v. 73, p. 97-100. 1988.

WATANABE, W. O.; LOSORDO, T. M.; FITZSIMMONS, K.; HANLEY, F. Tilápia production system in the americas: tecnological advances, trends, and challenges. Reviews in Fisheries Science [on line], v. 10, n. 384, p. 465-598, 2003. 
ZANONI, M. A.; CAETANO FILHO, M.; LEONHARDT, J.H. nilo (Oreochromis niloticus Linnaeus, 1757) em gaiolas. Animal Performance de crescimento de diferentes linhas de tilápia-do- $\quad$ Science, v. 22, n. 3, p. 683-687, 2000.

Protocolado em: 6 ago. 2007. Aceito em: 11 maio 2010. 